\title{
The positive impact of Medic Bleep, an asynchronous communication platform versus existing communication methods: an observational study
}

\author{
Authors: Meenakshi Jhala ${ }^{A}$ and Rahul Menon ${ }^{B}$
}

\section{Background}

Healthcare systems revolve around intricate relations between humans and technology. System efficiency depends on information exchange, which occurs on synchronous and asynchronous platforms. Traditional synchronous methods of communication may pose risks to workflow integrity and contribute to inefficient service delivery and medical care.

\section{Aim}

To compare synchronous methods of communication to Medic Bleep, an instant messaging (IM) asynchronous platform, and observe Medic Bleep's impact on clinical workflow, quality of work life, patient safety outcomes and hospital core operations.

\section{Methods}

Cohorts of healthcare professionals were followed using the time-motion study methodology over a 2-week period, using both the asynchronous platform Medic Bleep and synchronous methods like the non-cardiac pager. An 11-item questionnaire was sent out pre- and post-implementation of Medic Bleep and 24 interviews were conducted to identify staff attitudes towards both platforms.

\section{Results}

A statistically significant figure $(p<0.01)$ of 20.1 minutes' reduction in average task completion was seen with asynchronous communication, saving $58.8 \%$ of time when compared with traditional synchronous methods. For doctors, $p<0.0495$ and for nurses and midwives, $p<0.01$. A statistically significant reduction in mean task time was seen with 'to-takeout' (TTO), patient review, 'discharge and patient transfer' and 'escalation of care and procedure'. $67 \%$ of staff found implementation easy and a Likert scale value of 8.7 favoured asynchronous communication.

Authors: A St George's, University of London, London, UK; ' ${ }^{\text {Guy's }}$ and St Thomas' NHS Foundation Trust, London, UK

\section{Conclusion}

The asynchronous platform improved clinical communication compared to synchronous methods, contributing to efficiencies in workflow, and may positively affect patient care.

\section{Conflicts of interest}

None declared. 